\title{
Interlaboratory Study for Nickel Alloy 625 Made by Laser Powder Bed Fusion to Quantify Mechanical Property Variability
}

\author{
Christopher U. Brown, Gregor Jacob, Mark Stoudt, Shawn Moylan, John Slotwinski, and Alkan Donmez
}

(Submitted April 14, 2016; in revised form May 24, 2016; published online June 10, 2016)

\begin{abstract}
Six different organizations participated in this interlaboratory study to quantify the variability in the tensile properties of Inconel 625 specimens manufactured using laser powder bed fusion-additive manufacturing machines. The tensile specimens were heat treated and tensile tests were conducted until failure. The properties measured were yield strength, ultimate tensile strength, elastic modulus, and elongation. Statistical analysis revealed that between-participant variability for yield strength, ultimate tensile strength, and elastic modulus values were significantly higher (up to four times) than typical within-participant variations. Only between-participant and within-participant variability were both similar for elongation. A scanning electron microscope was used to examine one tensile specimen for fractography. The fracture surface does not have many secondary cracks or other features that would reduce the mechanical properties. In fact, the features largely consist of microvoid coalescence and are entirely consistent with ductile failure.
\end{abstract}

Keywords additive manufacturing, electron microscopy, interlaboratory study, mechanical behavior, round robin, selective laser melting, superalloys

\section{Introduction}

Metal-based additive manufacturing (AM) has great industrial potential. To realize this potential, AM processes must demonstrate the capability of consistently fabricating high quality industrial parts. However, the current level of maturity of this technology is not adequate to guarantee such consistency. Inter laboratory (round robin) studies with multiple participants are an important method for studying the robustness of AM processes and equipment to meet the industrial needs and requirements. Comparing the characteristics of parts made by different participants using the same set of AM process parameters provides a way to assess the variability of an AM process. Likewise, such collaborative studies using varying, but well defined, key AM process parameters can costeffectively improve the maturity of these processes by enabling better understanding of the effects of different parameters on the final part characteristics.

This round robin study is the third in a series of National Institute of Standards and Technology (NIST)-related round robin studies using laser powder bed fusion (LPBF) and metal

Christopher U. Brown, Gregor Jacob, Mark Stoudt, Shawn Moylan, and Alkan Donmez, National Institute of Standards and Technology, 100 Bureau Dr., Mailstop 8260, Gaithersburg, MD 20899, USA; and John Slotwinski, The Johns Hopkins University Applied Physics Laboratory, 11100 Johns Hopkins Road, Laurel, MD 20723, USA. Contact e-mail: christopher.brown@nist.gov.

powders (Ref 1). The first in the series of LPBF studies on metal powder used cobalt chromium powder ( $\mathrm{CoCr}$ ) (Ref 2). The variability between different round robin participants (between-participant variability) was significantly greater than the variability among the six tensile specimens on a single participant's build platform (within-participant variability). A second study conducted by an external group, Edison Welding Institute (EWI), on behalf of NIST used nickel alloy 625 (IN625) (Ref 3). The main goal was to develop and refine the manufacturing plan necessary to help control the AM machine parameters and develop design allowable seed data for a material's database.

The goal of this NIST round robin study is to quantify the variability in the tensile properties of Inconel 625 specimens manufactured by similar AM LPBF machines from different institutions and tested by NIST. IN625 is the powder chosen for this study because it is a readily available AM metal with clear industrial relevance. Although there are many test methods available to determine various mechanical properties of metals made using AM (Ref 4, 5), tensile tests were chosen for this study as a practical method to quantify variability.

This project is part of a NIST program to accelerate the use of AM parts in high performance applications in the US and part of a NIST developed roadmap for advancing AM (Ref 6). Although standards exist for conducting round robin, or interlaboratory, studies $(\operatorname{Ref} 7,8)$ in non-AM-specific fields, the AM field provides it's own unique set of challenges (Ref 1). As the AM round robin method improves, additional studies can then be conducted to provide quality data to an $\mathrm{AM}$ material database.

\section{Fabrication Procedure}

The focus of this study was to quantify the AM process variability from IN625 tensile specimens manufactured with multiple institutions LPBF machines from the same machine 
vendor. Six different organizations participated in the study: two universities, one commercial company, and three government institutions. Among the six participants, one had a newer model of the machine used by the others. In addition, another participant with a LBPF machine from a different vendor also built specimens for comparison purposes. The comparison of the resulting part characteristics from this machine with the others is part of a separate NIST internal report (Ref 9). The intent of the study was that all the AM machines would use the same process parameters and same build procedures to fabricate identical builds. However, because of differences in the models of the machines some machine settings were necessarily different. Participants with the same machine also had different levels of control to modify machine settings based on their purchased access levels and software versions.

Each participant fabricated one build consisting of six tensile specimens located horizontally on the build platform with their longitudinal axes parallel to the direction of the recoating arm motion (x direction) (Ref 10) and perpendicular to the build direction (see Fig. 1a). Figure 1b shows the final geometry of each tensile specimen which conforms to ASTM E8/E8M (Ref 9, 11). The tensile specimens were manufactured solidly connected to the build platform along the $15 \mathrm{~mm}$ length sections at each end. The solid connection helped anchor the specimen ends by preventing warping that could interfere with the recoater arm motion. The remaining middle section of the specimens has support structures underneath to provide stability and prevent warping due to residual stress. All participants used new build platforms (1045 steel, $25.4 \mathrm{~mm}$ thick, and $250 \mathrm{~mm} \times 250 \mathrm{~mm}$ ).

Typically, preparing to fabricate a part on an AM machine starts with a solid model. The solid model is converted to a tessellated model (six ".stl" files). The necessary support structures for the specimens are then added as tessellated models (six ".cli" files). The next step is to generate slice files corresponding to these tessellated files (twelve ".sli" files). All slice files are then combined in the "job file" to run the LPBF machine. NIST provided the job file to all participants. However, for participants not having the same version of machine software, NIST provided individual tessellated and slice files (Ref 9).

Along with the build files, NIST provided a manufacturing plan to each participant. The manufacturing plan consisted of a detailed list of steps for each participant to follow in order to fabricate the build. NIST requested that the participants track the machine parameters used in the process control document (PCD), part of the manufacturing plan. Some of the participants deviated from the manufacturing plan based on their level of control of their machines. Table 1 lists several key AM process parameters from the manufacturing plan. A separate NIST internal report (Ref 9) contains a copy of the manufacturing plan and process control document provided to each participant.

The PCD is a record, completed by the operator, of the machine parameter metadata. The PCD tracks settings and technical data before, during, and after the build. The PCD also provides the opportunity to correlate mechanical properties with machine settings. The PCD received from each participant is also included in the separate NIST internal report (Ref 9).

All nickel alloy (IN625) virgin powder needed for the study was purchased by NIST at one time from the same commercial lot and shipped in original $10 \mathrm{~kg}$ containers to participants. NIST was first to open the containers in order to take small samples for powder size distribution analysis. The chemical composition ( $\mathrm{wt} \%$ ) of the powder as reported by the powder supplier is: $\mathrm{N}$ ( $\geq 58), \mathrm{Cr}$ (20 to 23$)$, Mo (8 to 10$), \mathrm{Nb}$ (3.15 to $4.15), \mathrm{Fe}(\leq 5), \mathrm{Ti}(\leq 0.4), \mathrm{Al}(\leq 0.4), \mathrm{Co}(\leq 1), \mathrm{C}(\leq 0.1)$, $\mathrm{Ta}(\leq 0.05), \mathrm{Si}(\leq 0.5), \mathrm{Mn}(\leq 0.5), \mathrm{P}(\leq 0.015)$, and $\mathrm{S}$ $(\leq 0.015)$.

The powder supplier reported that the diameter of $98 \%$ of the powder was $53 \mu \mathrm{m}$ or less as determined by sieve analysis. We conducted our own size distribution analysis using sieves

Table 1 A brief selection of some nominal machine settings for the fabricated tensile bars

\begin{tabular}{ll}
\hline Parameter & \multicolumn{1}{c}{ Skin } \\
\hline Scan pattern & Striped \\
Feature size & $4 \mathrm{~mm}$ wide stripes \\
Laser power & $195 \mathrm{~W}$ \\
Scan speed & $800 \mathrm{~mm} / \mathrm{s}$ \\
Layer thickness & $20 \mu \mathrm{m}$ \\
Atmosphere & $\mathrm{N}_{2}$ \\
Raster line separation & $0.1 \mathrm{~mm}$
\end{tabular}

For a complete list of machine setting, see the NIST report (Ref 9) for the manufacturing plan

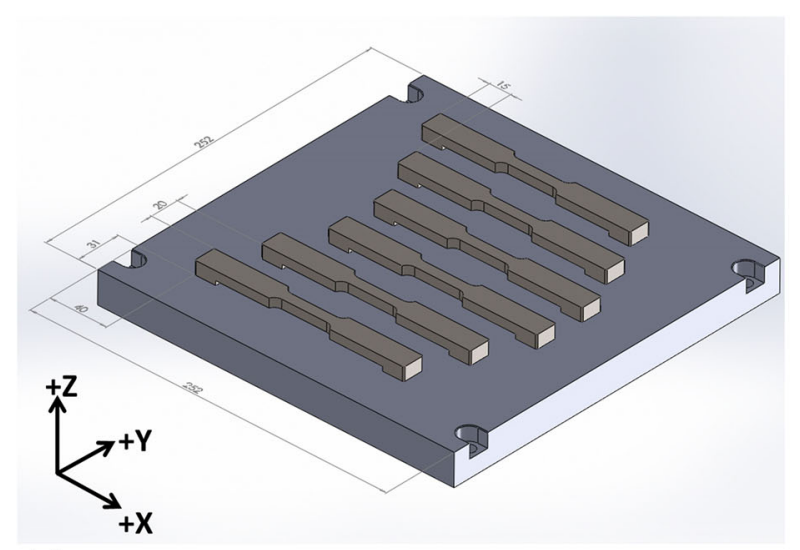

(a)

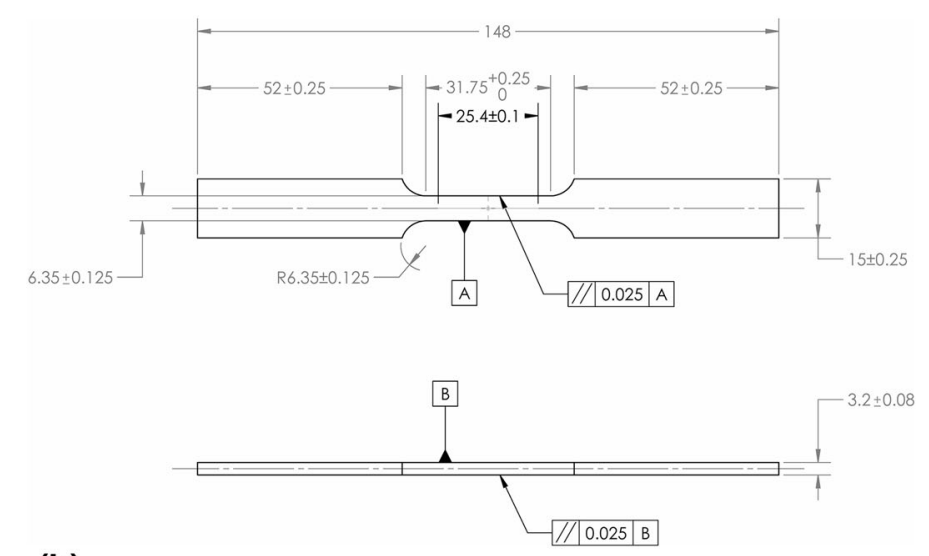

(b)

Fig. 1 (a) The build layout with six tensile bars. Dimensions are in mm's. (b) The final tensile specimen geometry with dimensions in mm's 
from powder samples from each participant container following ASTM B214 (Ref 12). The size distribution was D10= $18.46 \mu \mathrm{m} \pm 3.77 \mu \mathrm{m}, \mathrm{D} 50=34.32 \mu \mathrm{m} \pm 1.74 \mu \mathrm{m}$, and D90 $=$ $49.95 \mu \mathrm{m} \pm 1.08 \mu \mathrm{m}$ (Ref 9).

The participants shipped the completed build platforms, with tensile specimens still attached, back to NIST. NIST performed all necessary heat treatment and post machining to ensure consistency. Once received from the participants, the build platforms (with tensile specimens) were wrapped in a stainless steel bag and heat treated in an oven in an oxygen environment. The oven heated the build platform to $840^{\circ} \mathrm{C}$ in 6 hours and the temperature was held at $840{ }^{\circ} \mathrm{C}$ for 1 hour. The build platforms were then removed from the oven, unwrapped and removed from the stainless steel bag, and placed in front of a fan for rapid cooling. Wire electrical discharge machining (EDM) separated the specimens from the build platform and machined them to their final geometry.

\section{Evaluation of Mechanical Properties}

Before loading the tensile specimens for testing, each specimen was (1) visually examined to be sure that it did not have any macro flaws and (2) measured using a handheld micrometer to check its dimensions. Two parallel lines, $25 \mathrm{~mm}$ apart, were also drawn on the gage section. These lines were used to measure the elongation after fracture and were also a guide for placing the extensometer during the mechanical testing. The specimens placed in a tensile testing machine were tested until failure as per ASTM E8/E8M (Ref 11). Strain control from the extensometer was used during the initial loading at a strain rate of $0.015 \mathrm{~min}^{-1}$. Once the specimen was loaded past yielding, the extensometer was removed and the test continued until failure in displacement control at a strain rate $0.05 \mathrm{~min}^{-1}$. The fracture for all specimens occurred within the gage section as expected.

The fracture surface from one specimen from each participant was protected to carry out fracture surface analysis. A

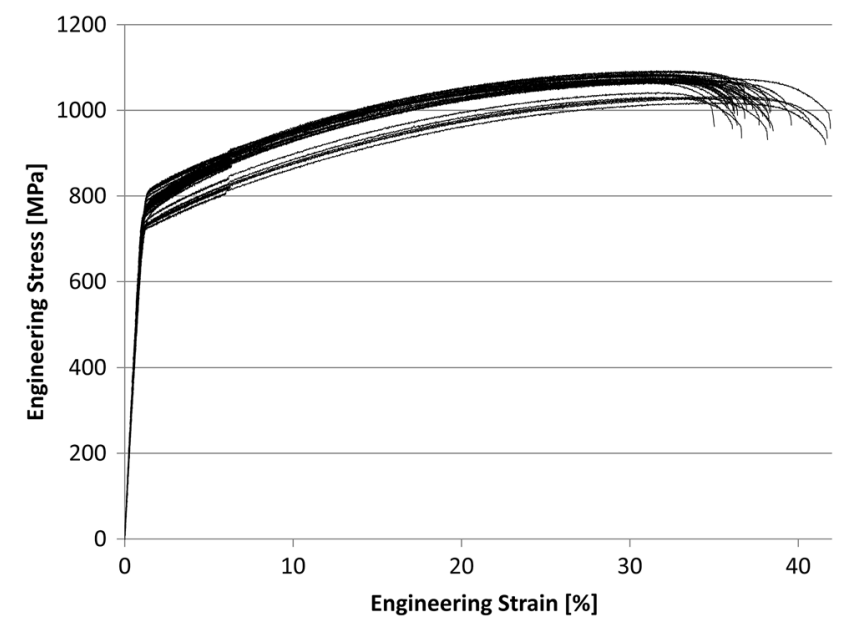

Fig. 2 Engineering stress vs. engineering strain for the 6 participants. The lower group of curves belong to Participant 4 as can also be seen in the results for $0.2 \%$ offset yield strength and ultimate tensile strength. The consistent step increase in engineering stress in all curves after an engineering strain of about $5 \%$ was the result of the increase in strain rate during the tensile test scanning electron microscope imaged the fracture surfaces at various levels of magnification.

The ultimate tensile strength (UTS), the elastic modulus $(E)$, and the $0.2 \%$ offset yield strength (YS) were determined from the stress-strain curves obtained from the measurements (see Fig. 2). Elongation $(A)$ was measured by carefully putting the tensile specimen back together after fracture and measuring the elongation of the $25 \mathrm{~mm}$ gage section.

\section{Results}

The yield strength, ultimate tensile strength, elastic modulus, and elongation, were measured for all (six) tensile specimens produced by each participant. Figure 3 summarizes the mechanical property results in the form of box plots. Each rectangular box comprises the middlemost $50 \%$ of the data, the thick horizontal line within the box marks the median, and the whiskers, if present, extend to the extreme data points. Circles indicate potential outliers: values that lie farther than 1.5 times the inter-quartile range from either the top or bottom sides of a box.

The mechanical properties from the tensile specimens were compared to the typical value and typical minimum value as quoted by the powder/LPBF machine provider. These are included in Fig. 4, 5, 6, and 7 as a red line for heat-treated (HT) specimens. A dashed red line represents the minimum value of the mechanical property if reported. Standard deviation bars are also included in these plots for each participant.

We performed a statistical comparison of data for each metric using Cochran's Q test (Ref 13). Statistical analysis revealed that between-participant variability for YS, UTS, and E values was significantly higher (up to four times) than typical within-participant variations. Only the A value variability was similar for both between-participant and within-participant. Most of the measured values were above the typical values for the alloy as quoted by the material supplier.

\section{Fractography Analysis}

After failure, the fracture surfaces of one random tensile bar from each participant were reserved for analysis in a scanning electron microscope (SEM). That is, these tensile bars were not measured for elongation, since the elongation measurement requires fitting the two fractured surfaces together. Reserving these specimens preserved the delicate features on the fracture surface and reduced the likelihood of further contamination. The results of the fractographic analyses conducted on one specimen are described here.

Fractographic analysis was performed on tensile specimen 6 from participant 5. The tensile specimen in the following images was annealed after fabrication. The fracture surface of this specimen does not appear to have many secondary cracks or other features that would reduce the mechanical properties (Fig. 8,9). In fact, the features exhibited on the fracture surface largely consist of microvoid coalescence and are entirely consistent with ductile failure. Some faceting is present, which could reflect some localized transgranular cracking (shown in Fig. 9, region 1), but this was not reflected in the mechanical data for this specimen. At higher magnification in Fig. 9 

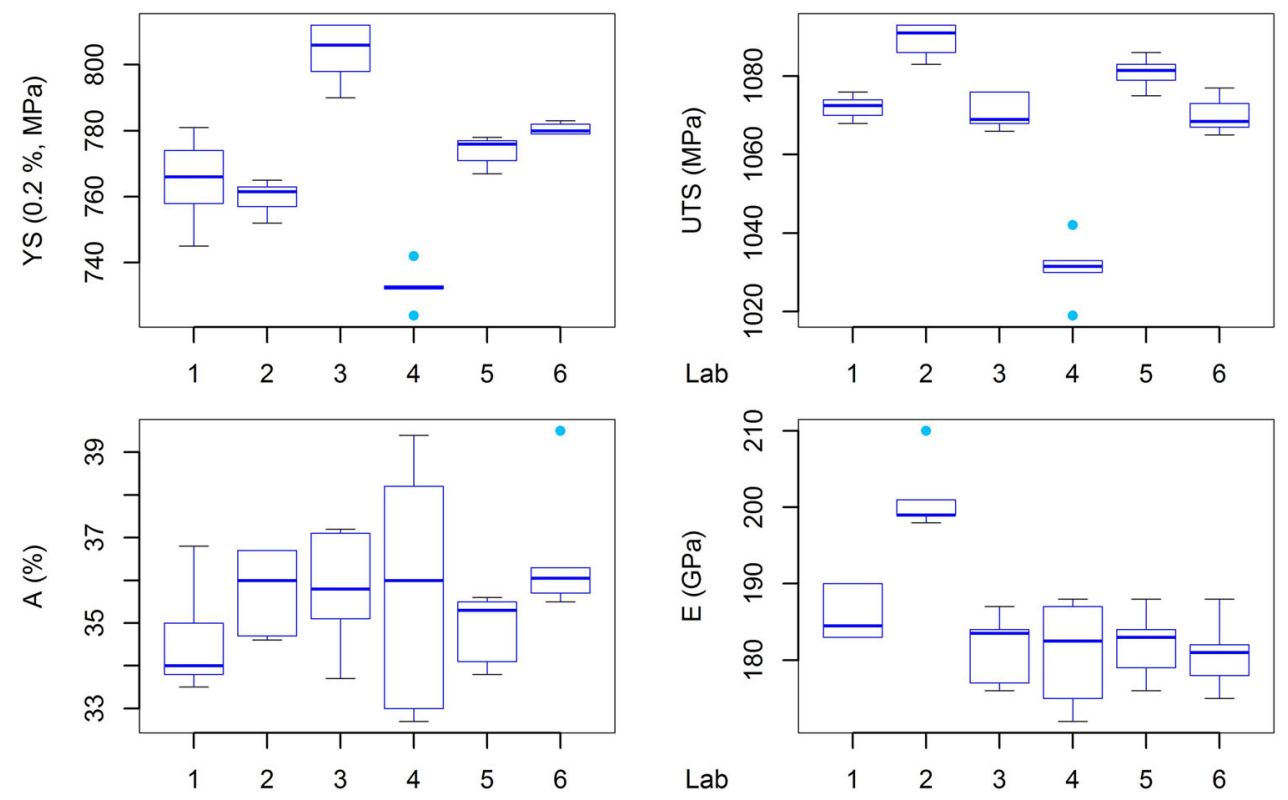

Fig. 3 Box plots of measured mechanical properties from the 6 participants

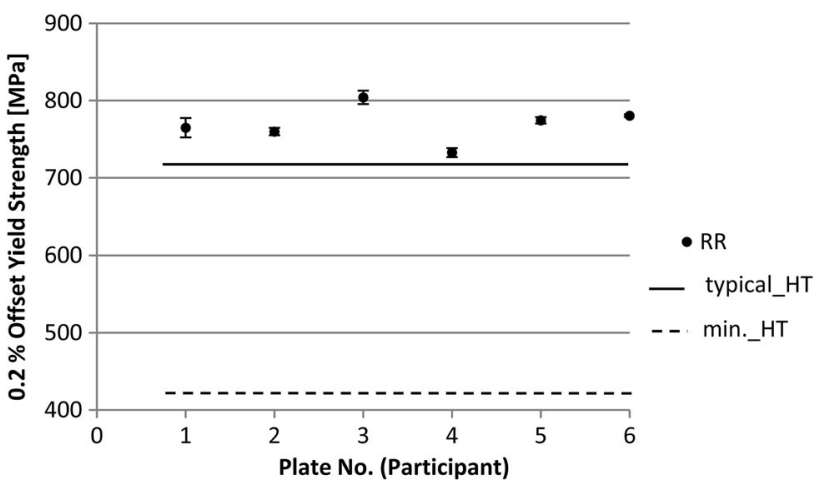

Fig. $40.2 \%$ offset yield strength was higher than the typical value quoted by the powder/LPBF machine supplier for specimens

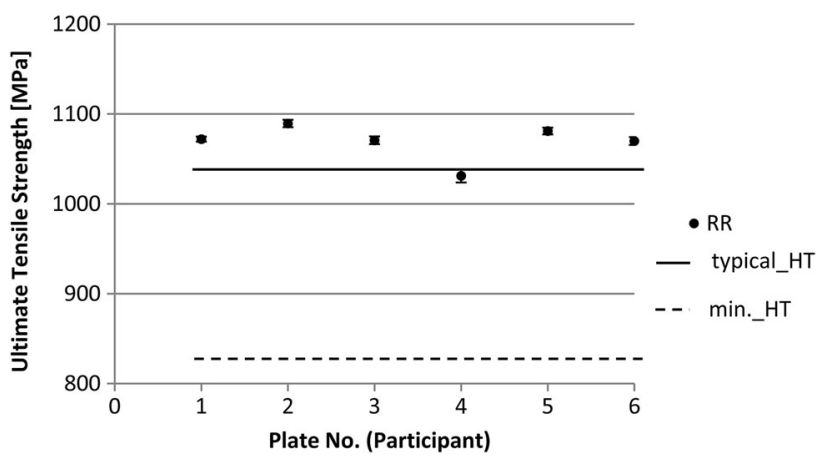

Fig. 5 Ultimate tensile strength was above and below the typical heat-treated value as reported by the powder/LBPF machine supplier, however all results from this study were well above the minimum heat-treated value

$(\times 140)$ horizontal secondary cracks and steps can be seen parallel to the build direction that may indicate a weaker interlayer interface. In addition, the surface exhibits a ductile

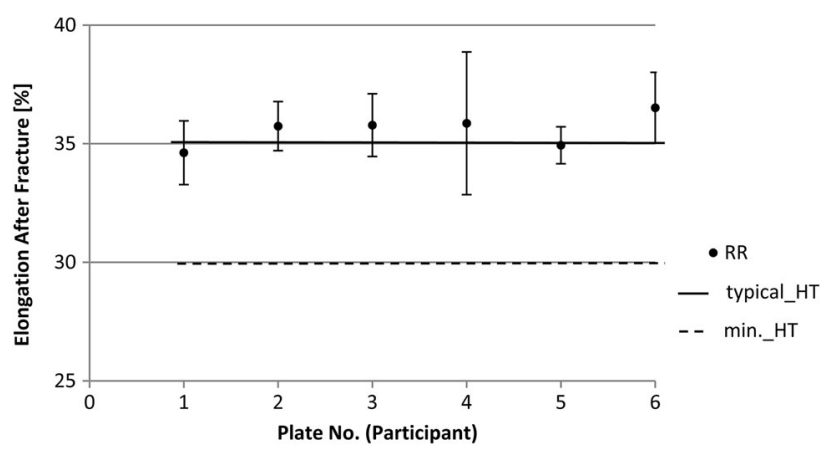

Fig. 6 Elongation was not significantly different among the participants

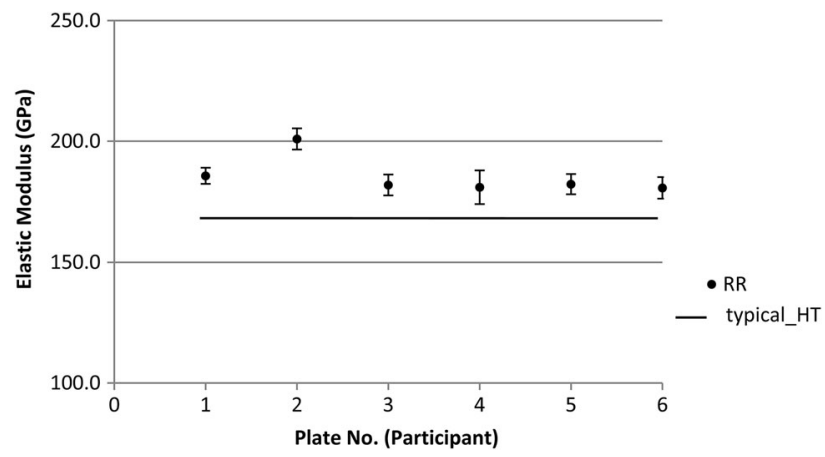

Fig. 7 Elastic modulus among the participants was significantly different

hinge, which was likely part of the final fracture (bottom left of Fig. 8).

Figure 10 is a higher magnification view $(\times 1000)$ of the fracture surface showing the fine-scaled dendrites resulting 


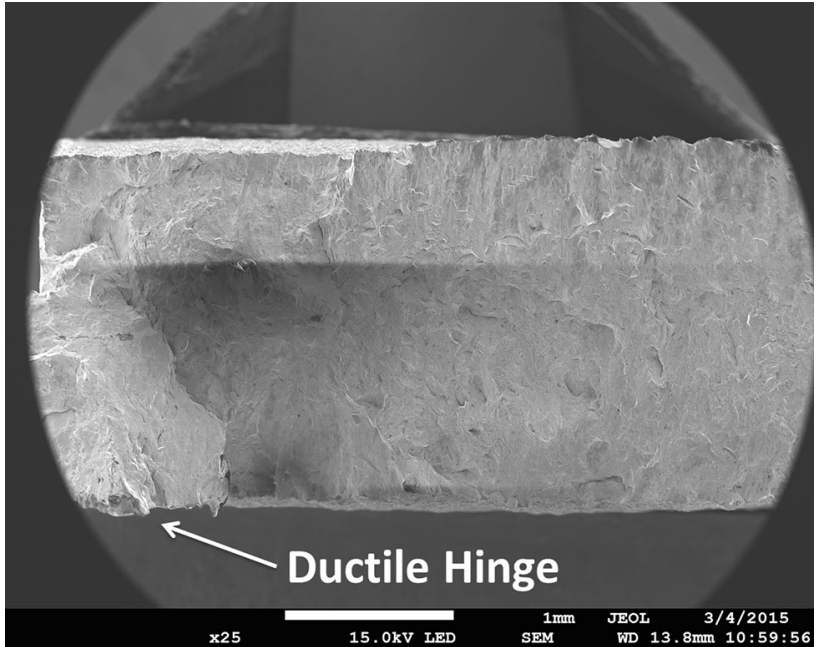

Fig. 8 A low magnification view $(\times 25)$ of the tensile specimen 6 cross section showing a ductile hinge at the bottom left, (i.e., a raised section of the fracture surface that was the last contact point between the two fracture surfaces as they separated)

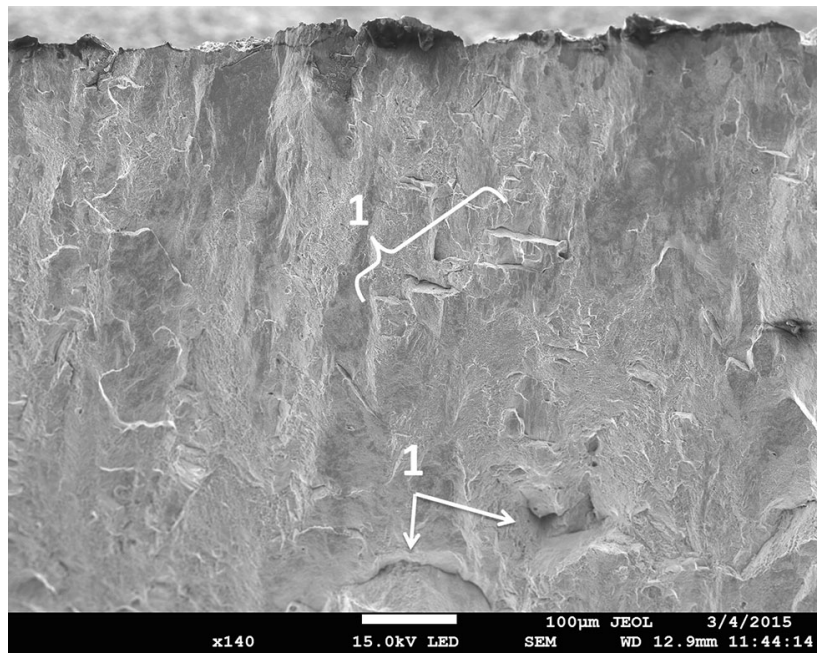

Fig. 9 A higher magnification view $(\times 140)$ of the fracture surface from specimen 6 , participant 5 , showing several large transgranular cleave-like (TCL) facets (1), suggesting that some localized cracking might have occurred during this test. Some of these facets appear to be parallel to the build direction while others are not

from micro-segregation during the build process (region 1). While some evidence of localized cracking is again observable (e.g., the transgranular cleavage-like features shown as region 2 , and the small secondary crack shown as region 3 ), the overall morphology of this surface is consistent with ductile failure. No un-melted or partially melted particles were observed within cracks; however, inclusions were observed on the surface (region 4).

Figure 11 is a high magnification view $(\times 8000)$ of the finescaled dendritic segregation seen on the fracture surface. Since the growth direction of the dendrites is into the page, it is likely that decohesion along dendrite interfaces was the dominant factor in the fracture of this specimen. Figure 12, 13, 14, 15, 16 , and 17 show additional images.

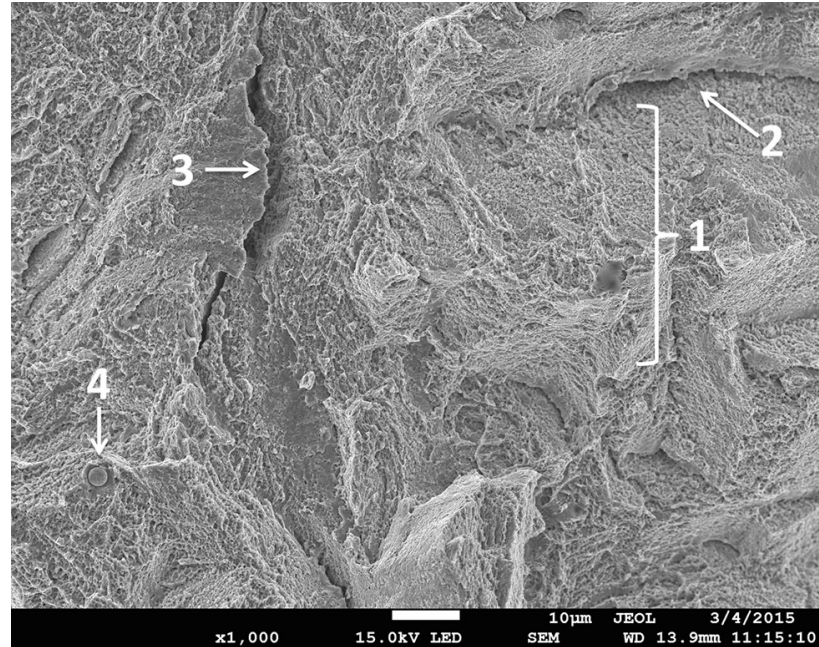

Fig. 10 A high magnification image $(\times 1000)$ of the fracture surface shows primarily dendrites (1) with some possible transgranular cleavage-like features (2). A small secondary crack (3) and an inclusion are also present on the surface (4), but were not significant factors in the primary fracture

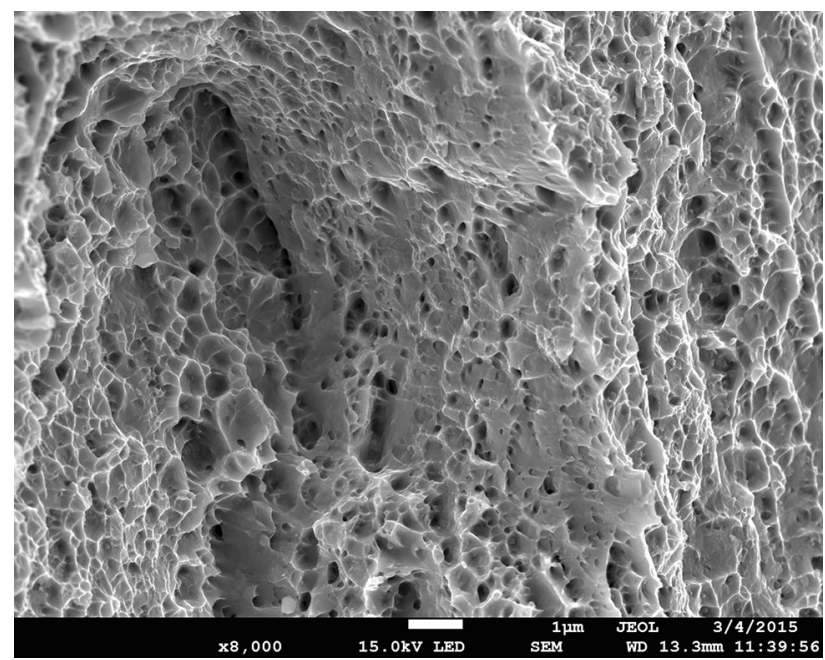

Fig. 11 A high magnification image $(\times 8000)$ of the fine-scaled dendrites on the fracture surface

\section{Discussion}

The primary goal of this study was to characterize the interlaboratory variability of nickel alloy 625 tensile properties from specimens produced by similar AM LPBF machines from different institutions. Results from this study agree with previous studies that have documented interlaboratory variability through round robin studies (Ref 2). However, there were drawbacks to this study. It was our intent to have all the participants follow the manufacturing plan exactly so that every build was fabricated the same way. However, due to different AM LPBF machines, different build software versions, and participant's different levels of access and control over their software and AM machines, the build process for each participant was not exactly the same. Some participants used 


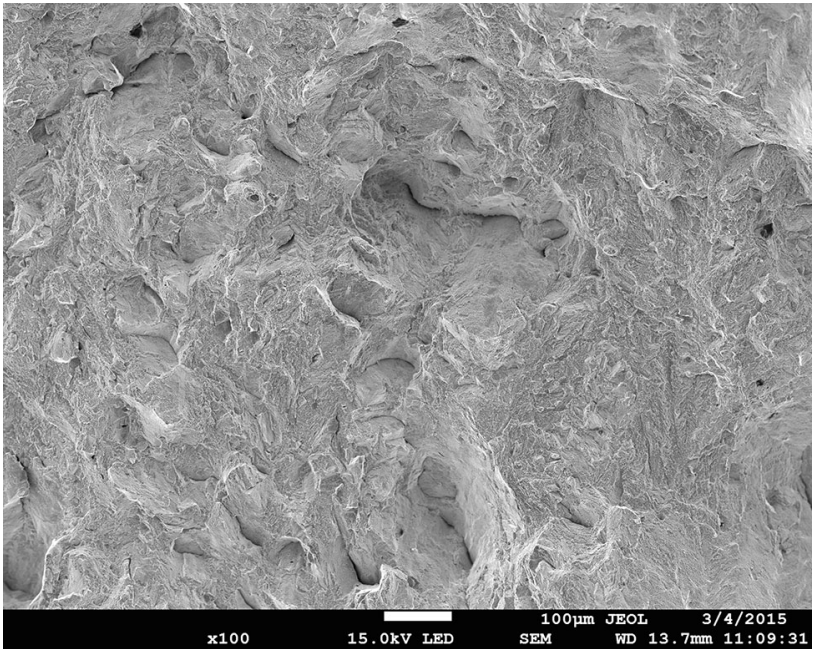

Fig. 12 A higher magnification $(\times 100)$ of a region of Fig. 8 that does not appear to have horizontal secondary cracks related to the build layer but rather localized transgranular cracking in various directions

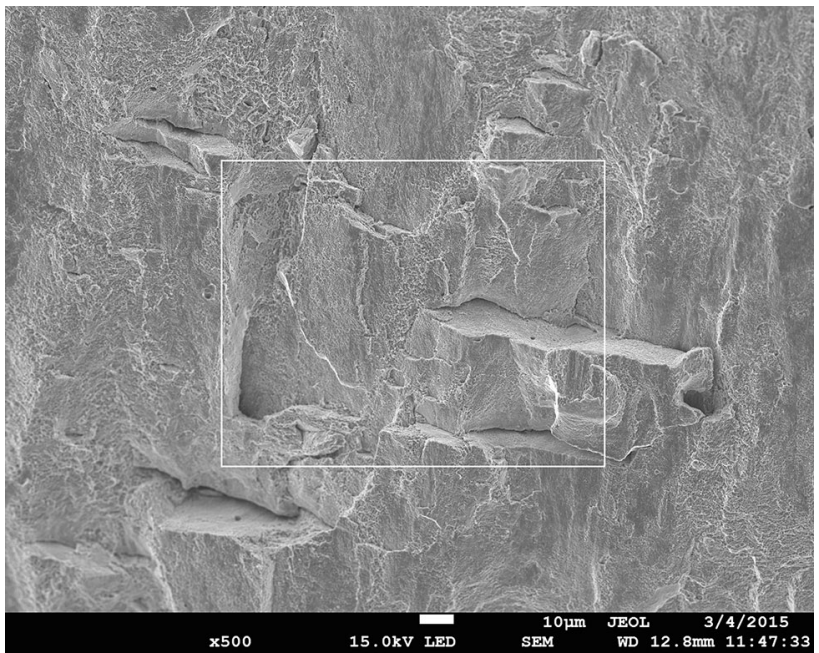

Fig. 13 A higher magnification $(\times 500)$ of the top group of facets labeled in Fig. 9. The white box is the region magnified in Fig. 14. These step-like cracks align with the layered build direction

different LPBF machine settings that may have affected the mechanical properties of the tensile specimens and thus affected the variability of the results.

We became aware of the different machine settings when we analyzed the process control documents returned from each participant. As an extension to our study, we analyzed the differences among the settings to determine if these differences contributed to the statistical variability of the mechanical properties of the test specimens. The machine settings we chose to examine were: energy density $\left(\mathrm{J} / \mathrm{mm}^{3}\right)$, volume rate $\left(\mathrm{mm}^{3} / \mathrm{s}\right)$, layer thickness, and laser power. Volume rate is the measure of laser speed $(\mathrm{mm} / \mathrm{s})$ for a hatch distance $(\mathrm{mm})$ with a specific powder layer thickness ( $\mathrm{mm})$. Energy density is the laser power (W or $\mathrm{J} / \mathrm{s}$ ) per the volume rate $\left(\mathrm{mm}^{3} / \mathrm{s}\right.$ ). Changes in settings did not induce statistically significant changes in the mechanical properties whose values we measured. We verified this by fitting mixed effects models (Ref 14) to the values of the

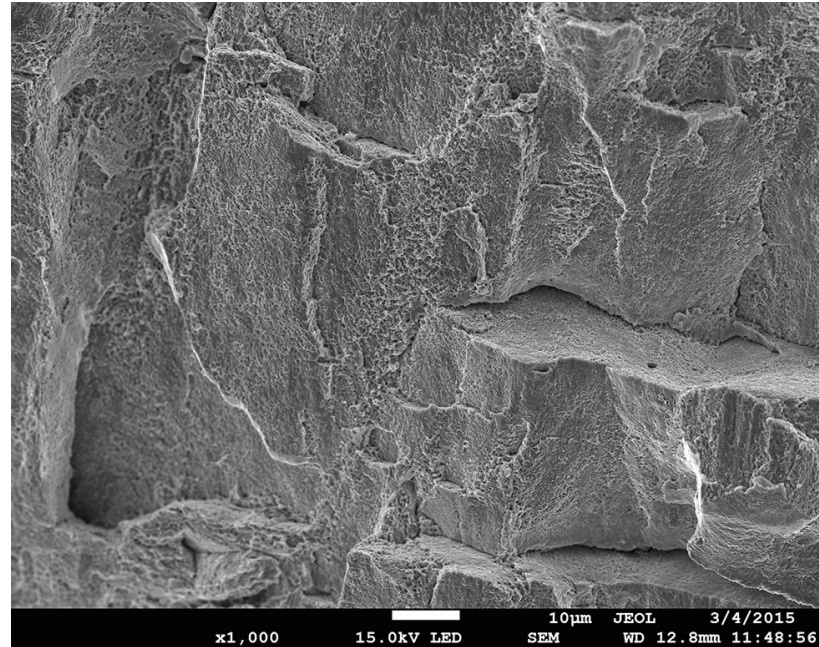

Fig. 14 Several transgranular cleave-like facets on the fracture surface

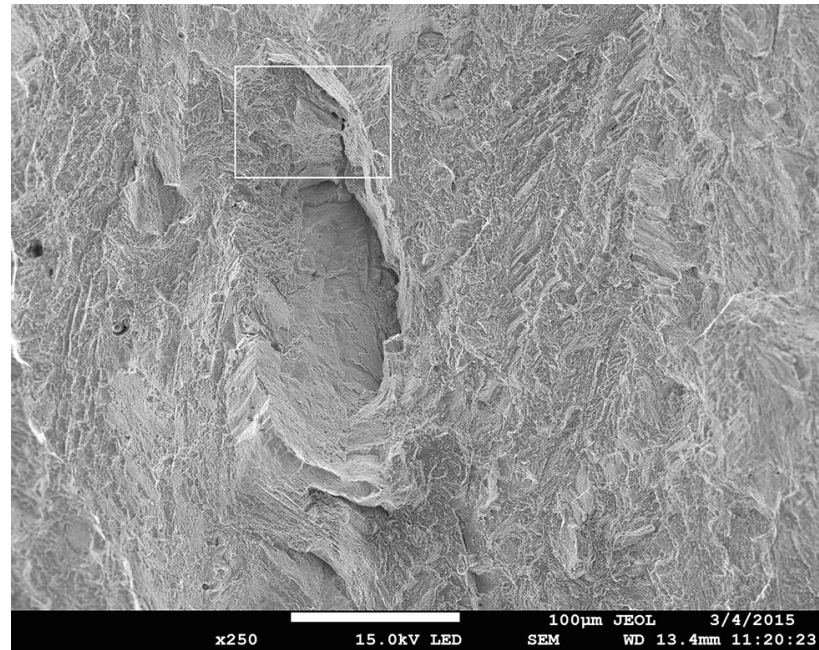

Fig. 15 A different area of the fracture surface then Fig. 10 but with similar magnification $(\times 250)$. A large TCL facet is in the middle of the image and the top of the facet was magnified in Fig. 16

mechanical properties, with laboratory as random effect and each of the settings as fixed effect, and found that the fixed effect was not statistically different from zero. This does not mean that there isn't a relationship, only that based on the data gathered in this study, we were not able to make a statistically significant conclusion.

The work in this study, as currently designed, clearly demonstrates the variability in the data, but makes it difficult to attribute the variability to a specific cause. A typical round robin study is designed to be simple and hold constant as many variables as possible. We ended up with a range of different machine settings, but that was not very broad since the experiment setup was such that the settings were not supposed to be different. In order to determine the relationship between machine settings and mechanical properties, a specifically designed experiment should be conducted for addressing this goal. The results of the current study indicate that in such an experiment, varying the laboratory would be an important 


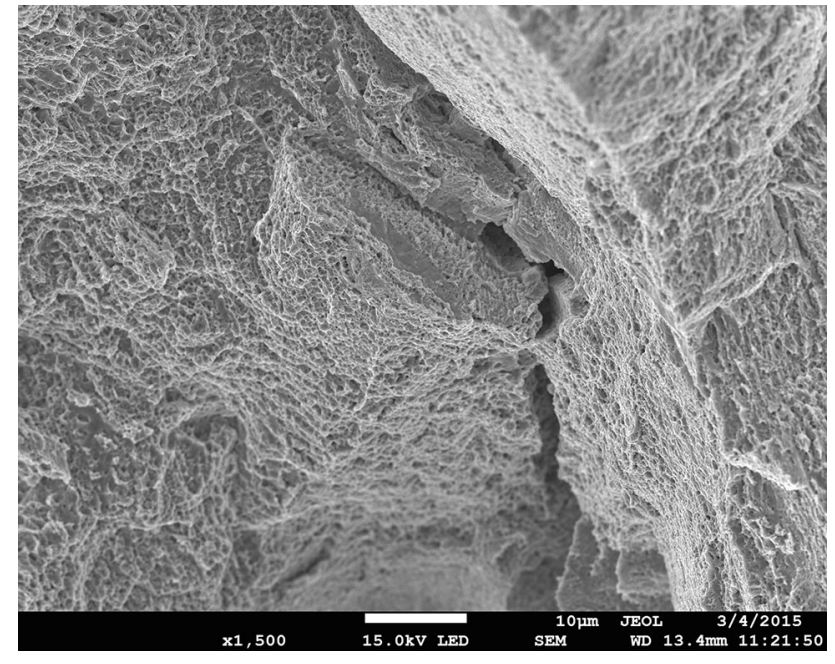

Fig. 16 Dendrites are evident around the secondary crack

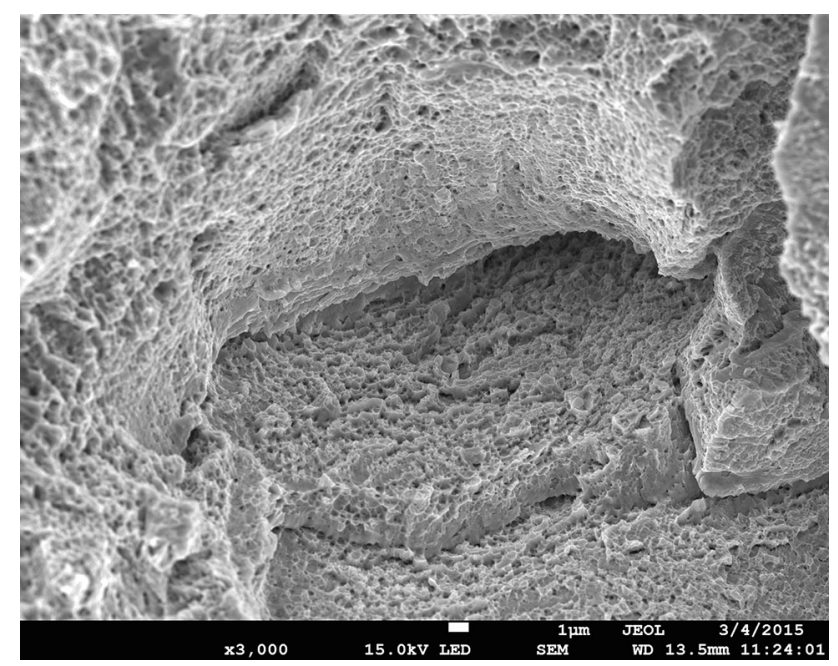

Fig. 17 A high magnification image $(\times 3000)$ of the fracture surface shows primarily dendrites with a transgranular cleavage-like feature

factor to include, but there are several additional factors to be included in the study and varied deliberately. In summary, we were not looking for a link between machine settings and mechanical properties. However, because we ended up having a small range of settings we tried to see if we could detect a relationship with the data that we had, but we could not. A better experiment to test for these relationships is being designed.

As we look forward to conducting future round robin studies and ultimately toward generating data for a materials property database, we find several takeaways from this study that will be helpful. One issue was that the manufacturing plan and process control document were difficult to translate exactly when the software versions were different, access was limited to AM machine to make changes, or the AM machine was different. A lesson learned from this study therefore is that our manufacturing plan and process control document need to be either machine/software agnostic or provide separate, more specific plans for each machine/software version to help ensure that the participants can follow the plan. This will require additional input during planning stages of the study to develop machineor software-specific plans if necessary.

The following is a list of recommendations for future round robin studies:

- Increasing the number of participants would be statistically helpful, rather than increasing the number of test coupons made by each participant.

- Alter our manufacturing plan and process control document to reflect the participant's AM software and machine to help reduce any changes in machine settings.

- Future studies will investigate the effects of changing specific machine settings on the mechanical properties of the test coupons from participants.

- Add several witness blocks to each build platform adjacent to the test coupons. The blocks will help determine build characteristics from the AM machine before the stress relief heat treatment is applied.

\section{Conclusions}

- This study demonstrated between-participant variability in the mechanical property data, but we could not determine the cause of that variability from this study. Our results indicate that varying the laboratory is an important factor to include in a follow-up study. There are also several additional factors such as machine settings that we could vary deliberately to try to determine the source of mechanical property variability.

- Between-participant variability for yield strength, ultimate tensile strength, and elastic modulus values were significantly higher (up to 4 times) than typical within-participant variations. Between-participant and within-participant variability were both similar for elongation.

- A scanning electron microscope was used to examine the tensile bar fracture surface which did not have many secondary cracks or other features that would reduce the mechanical properties. The fracture features largely consist of microvoid coalescence and are consistent with ductile failure.

- The manufacturing plan and process control document need to be either machine/software agnostic, or specific plans need to be issued for each machine/software version in a round robin study to help ensure that the participants can follow the plan exactly.

\section{Acknowledgment}

The authors would like to thank the following researchers: Antonio Possolo for his statistical analysis; Stephanie Watson, Max Peltz, and Clarissa Ferraris for their powder size analysis. The authors would also like to thank the other participants in this study including GE Global Research (Prabhjot Singh, John Deaton, Laura Dial), Honeywell Aerospace (Donald Godfrey, Bob Rasmussen, Brian Baughman), Arizona Commerce Authority (Dan Franklin), University of Louisville (Tim Gornet, Brent Stucker, Gary Graf, Matt Taylor), Western Illinois University-Quad City 
Manufacturing Lab (Eric Faierson), Picatinny Arsenal (Stacey Kerwien, Elias Jelis, Matthew Clemente, Dave Dekmar, Sr.), Aberdeen Proving Ground (Rick Moore, Lester Hitch).

\section{Disclaimer}

Certain commercial entities, equipment, or materials may be identified in this document in order to describe an experimental procedure or concept adequately. Such identification is neither intended to imply recommendation or endorsement by the National Institute of Standards and Technology, nor is it intended to imply that the entities, materials, or equipment are necessarily the best available for the purpose.

\section{References}

1. S. Moylan, C.U. Brown, and J. Slotwinski, Recommended Protocol for Round-Robin Studies in Additive Manufacturing, ASTM J. Test. Eval., 2016, 44(2), p 1009-1018

2. J.A. Slotwinski, W.E. Luecke, E.A. Lass, A. Possolo, Interlaboratory Mechanical-Property Study for Cobalt-Chromium Alloy Made by Laser Powder-Bed-Fusion Additive Manufacturing, J. Test. Eval. (in press)

3. S.M. Kelly, Volume 1: Development and Measurement Analysis of Design Data for Laser Powder Bed Fusion Additive Manufacturing of Nickel Alloy 625 Final Technical Report, EWI, Columbus, OH, 2014
4. J. Slotwinski, A. Cooke, S. Moylan, Mechanical Property Testing for Metal Parts Made via Additive Manufacturing: A Review of the State of the Art of Mechanical Property Testing, National Institute of Standards and Technology Internal Report 7847, 2012

5. Standard Guide for Evaluating Mechanical Properties of Metal Materials Made via Additive Manufacturing Processes, F3122-14, ASTM International, 2014

6. Measurement Science Roadmap for Metal-Based Additive Manufacturing, prepared by Energetics Inc. for the National Institute of Standards and Technology, 2013

7. Standard Guide for Conducting an Interlaboratory Study to Determine the Precision of a Test Method, D7778-15, ASTM International, 2015

8. Standard Practice for Conducting an Interlaboratory Study to Determine the Precision of a Test Method, E691-15, ASTM International, 2015

9. C.U. Brown, G. Jacob, M. Stoudt, A. Possolo, S. Moylan, and A. Donmez, Supplementary information for an interlaboratory study of additively manufactured nickel alloy (IN625) tensile specimens, National Institute of Standards and Technology Internal Report 8100, 2016

10. Standard Terminology for Additive Manufacturing - Coordinate Systems and Test Methodologies, ISO/ASTM 52921:2013(E), 2013

11. Standard Test Method for Tension Testing of Metallic Materials, E8/ E8M-15a, ASTM International, 2015

12. Standard Test Method for Sieve Analysis of Metal Powders, B214-15, ASTM International, 2015

13. L.V. Hedges and I. Olkin, Statistical Methods for Meta-Analysis, Academic Press, San Diego, 1985

14. J.C. Pinheiro and D.M. Bates, Mixed-Effects Models in S and S-Plus, Springer, New York, 2000 